\title{
Momordica charantia for type 2 diabetes mellitus.
}

\begin{abstract}
Momordica charantia (bitter gourd) is not only a nutritious vegetable but it is also used in traditional medical practices to treat type 2 diabetes mellitus. Experimental studies with animals and humans suggested that the vegetable has a possible role in glycaemic control. To assess the effects of mormodica charantia for type 2 diabetes mellitus. Several electronic databases were searched, among these were The Cochrane Library (Issue 1, 2012), MEDLINE, EMBASE, CINAHL, SIGLE and LILACS (all up to February 2012), combined with handsearches. No language restriction was used. We included randomised controlled trials (RCTs) that compared momordica charantia with placebo or a control intervention, with or without pharmacological or non-pharmacological interventions. Two authors independently extracted data. Risk of bias of the trials was evaluated using the parameters of randomisation, allocation concealment, blinding, completeness of outcome data, selective reporting and other potential sources of bias. A meta-analysis was not performed given the quality of data and the variability of preparations of momordica charantia used in the interventions (no similar preparation was tested twice). Four randomised controlled trials with up to three months duration and investigating 479 participants met the inclusion criteria. Risk of bias of these trials (only two studies were published as a full peer-reviewed publication) was generally high. Two RCTs compared the effects of preparations from different parts of the momordica charantia plant with placebo on glycaemic control in type 2 diabetes mellitus. There was no statistically significant difference in the glycaemic control with momordica charantia preparations compared to placebo. When momordica charantia was compared to metformin or glibenclamide, there was also no significant change in reliable parameters of glycaemic control. No serious adverse effects were reported in any trial. No trial investigated death from any cause, morbidity, health-related quality of life or costs. There is insufficient evidence on the effects of momordica charantia for type 2 diabetes mellitus. Further studies are therefore required to address the issues of standardization and the quality control of preparations. For medical nutritional therapy, further observational trials evaluating the effects of momordica charantia are needed before RCTs are established to guide any recommendations in clinical practice.
\end{abstract}

Keyword: Type 2 diabetes mellitus; Momordica charantia. 